\title{
Dynamics of Soliton-Like Excitations in a Chain of a Polymer Crystal: Influence of Neighbouring Chains Mobility
}

\author{
Elena $A Z U B O V A A^{\dagger}$ and $N K B A L A B A E V^{\ddagger}$ \\ $\dagger$ N N Semenov Institute of Chemical Physics, Russian Academy of Sciences \\ Moscow, Russia \\ E-mail: zubova@center.chph.ras.ru \\ $\ddagger$ Institute of Mathematical Problems of Biology, Russian Academy of Sciences \\ Pushchino, Russia
}

\begin{abstract}
We investigate influence of mobility of neighbouring chains on dynamics of soliton-like excitations in a chain of the simplest polymer crystal (polyethylene in the "united atoms" approximation) using molecular dynamics simulation. We present results for point-like structural defects: static and moving at low, medium and high velocities; and examine how the structure of a crystal will affect them.
\end{abstract}

\section{Introduction}

The present work is concerned with the behaviour of a system presumed to be close to an integrable one, namely Sine-Gordon system. Sine-Gordon equation is well known to be the simplest continual one-dimensional model of a dislocation in a crystal (the well known Frenkel-Kontorova model). It can be equally used to describe nonlinear dynamics of a directed macromolecular chain subjected to a periodic potential $[1,2]$. Because it is a periodic potential which is generated by immobile neighbouring chains in a polymer crystal, it was supposed that one can identify topological solitons of Sine-Gordon type with point defects in a polymer chain (vacancies and inclusions) which are caused by local deformations of the chain without breaking of intrachain bonds.

The notion of soliton-type mobility of vacancies in polymer crystal opposed to diffusiontype one in crystal of low-molecular substances first arose in attempting to explain anomalously rapid dielectric relaxation of oxidized polyethylene. They speculated that it is topological solitons propagating along the chains and changing the state of the chains after their passing which provide such a rapid relaxation. There appeared many physical works employing the idea of soliton-type mobility of point defects in polymer crystals (see reviews $[3,4,5])$.

But one can easily see a very weak point in the theory: mobility of neighbouring chains in a real crystal which has hence much more degrees of freedom than one chain subjected to a potential. Mobility of neighbouring chains must influence dynamics of defects in the chain. 
To find out the character of the influence, Noid et. al. [6] have carried out a moleculardynamics simulation of motion of a defect in a chain of the simplest polymer crystal (polyethylene in the united atoms approximation). They have claimed its nonsoliton nature: defects stopped after passing of several tens of periods of the chain. So it looked like the end of soliton ideas in polymer physics; and facts of anomalously rapid relaxation in polymers became the more puzzling one.

To approach the real physical situation one have to find out which alterations in an integrable system can change so drastically dynamics of soliton-like excitations and which can not. Two types of changes have been already investigated: discreteness of the chain by Peyrard and Kruskal [7] and variation of the form of the potential out of sine function by Peyrard and Remoisset [8]. The situation in PE crystal at not extremely high soliton velocities is unrelated to these types of changes. Two other possibilities have been left absolutely unstudied (except above-mentioned work [6]): mobility of neighbouring chains and structure of a crystal. They are objects of the present work.

\section{Numerical model of a polymer crystal}

We have taken the following model for a polymer crystal [9] (polyethylene with collective atoms; see Fig. 1): the chains consist of plane trans-zigzags; the bonds between the atoms (point particles of mass $m$ ) are absolutely rigid of length $l_{0}$; the deformation energies of the valence $\left(\theta_{n}\right)$ and conformational $\left(\phi_{n}\right)$ angles are given by

$$
\begin{aligned}
& U_{3}\left(\theta_{n}\right)=\frac{1}{2} K_{\theta}\left(\theta_{n}-\theta_{0}\right)^{2}, \\
& U_{4}\left(\phi_{n}\right)=\alpha+\beta \cos \left(\phi_{n}\right)+\gamma \cos \left(3 \phi_{n}\right),
\end{aligned}
$$

and, atoms separated by more than two neighbours or belonging to different chains interact through the potential

$$
U(r)= \begin{cases}U_{L J}(r)-U_{L J}(R), & r \leq R \\ 0, & r>R\end{cases}
$$

where $U_{L J}(r)=4 \epsilon\left((\sigma / r)^{12}-(\sigma / r)^{6}\right)$ is the Lennard-Jones potential with a minimum at $r_{0}=2^{1 / 6} \sigma$. The numerical values of the constants employed here are listed in Table 1.

Table 1. Parameters of the model crystal

\begin{tabular}{|c|c|c||c|c|c|}
\hline parameter & value & ref. & parameter & value & ref. \\
\hline$m$ & 14 a.m.u. & - & $\beta$ & $1.675 \mathrm{~kJ} /$ mole & 10 \\
$l_{0}$ & $1.53 \AA$ & 10 & $\gamma$ & $6.695 \mathrm{~kJ} /$ mole & 10 \\
$\theta_{0}$ & $113^{\circ}$ & 10 & $\epsilon$ & $0.4937 \mathrm{~kJ} /$ mole & 11 \\
$K_{\theta}$ & $331.37 \mathrm{~kJ} /$ mole & 10 & $\sigma$ & $3.8 \AA$ & 11 \\
$\alpha$ & $8.370 \mathrm{~kJ} /$ mole & 10 & $R$ & $2 r_{0}$ & - \\
\hline
\end{tabular}

We have taken periodic boundary conditions for the crystal in all three directions. The cells of the computational grid were chosen to have the shape of a rectangular parallelepiped. The corresponding classical first order Lagrange equations were solved numerically using a Berle leap-frog algorithm [12] taking note of the limitations imposed by the rigid bonds [13]. 
Periodic boundary conditions make it possible to follow the soliton dynamics in the direction along the axis of the molecules for an unlimited time and to avoid introducing unphysical boundary conditions such as a rigidly attached second coordination sphere in the plane of the transverse cross section. In order to keep a soliton from interacting with itself, the number of molecules in the grid cell was chosen so that the image of each molecule lies no closer than its fourth coordination sphere, while the length of the molecule for a soliton extending on the order of 35 chain periods (c) was assumed to be $200 c$ (two $\mathrm{CH}_{2}$ groups fall within a single period).

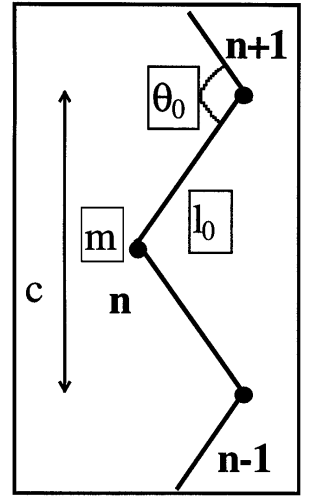

Figure 1. A chain of the simplest polymer crystal (polyethylene in "united atoms" approximation): chain parameters and local atomic coordinates.

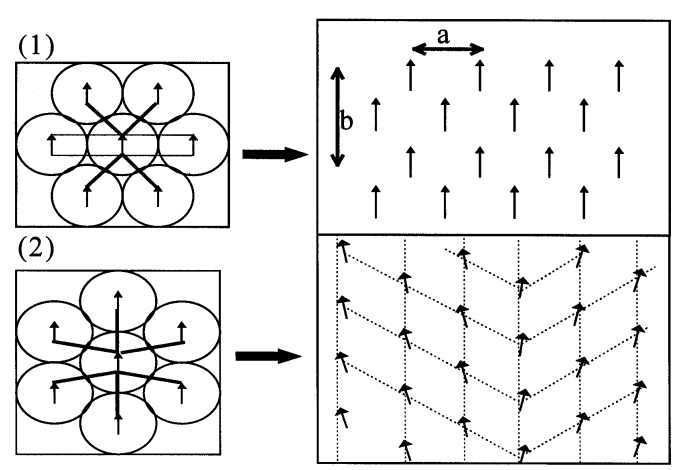

Figure 2. Packing of plane zigzags into a crystal: possible equilibrium configurations (1) stable and (2) unstable (shown here is the plane of the transverse cross section of the molecules; the arrows denote the direction from the nearest atom of the molecule under the plane to the nearest above the plane).

\section{Equilibrium crystal configuration of a polyethylene crystal in the united atom model}

Since the projected length of a molecule on its perpendicular cross sectional plane is $l_{\perp}=$ $0.843 \AA$, the van der Waals radius $r_{0}=4.265 \AA \approx 5 l_{\perp}$, the packing of the molecules in the crystal will be close to cylindrical. Two different (mechanically) equilibrium configurations are conceivable (Fig. 2). Both have a monoclinic cell and similar energies. The second, however, is unstable and stratifies into two domains, both of which correspond to the first configuration. The parameters a and $\mathrm{b}$ of the stable structure depend on the cutoff radius $R$. When $R=2 r_{0}$, equilibrium parameteres of crystal $a=3.980 \AA, b=7.966 \AA$. The period along the molecular axis is equal to $c=2.554 \AA$. The density of the sample is $\rho=1.155 \mathrm{~g} / \mathrm{cm}^{3}$.

Molecular-dynamics modelling of a polyethylene crystal in the united atom approximation has shown that there is no local minimum in the potential energy for an orthorhombic structure for any such cell parameters. The numerical simulation yields a minimum of this sort only for a model of polyethylene in which the $\mathrm{CH}_{2}$ group is modelled by three spatially separated force centers. Thus, an orthorhombic structure in polyethylene can exist only because of the presence of lateral groups, and not of the shape of the chain skeleton. 


\section{Theoretically expected character of the vacancy dynamics in a polymer crystal chain}

We shall show that the simplest description of the dynamics of a vacancy in a chain of a polymer crystal in the approximation of immobile neighbours can be reduced to a sine-Gordon equation for the transverse displacement $u$ of the atoms in the chain (see Fig. 1).

In fact, the effective substrate potential is obtained by calculating the crystal energy with all the molecules fixed but one, which moves along the axis. In the stable equilibrium configuration of the crystal it can be approximated to within two percent by two harmonics:

$$
V(u)=A\left[1-\cos \left(\frac{2 \pi}{c} u\right)\right] .
$$

with $A=0.274 \mathrm{~kJ} /$ mole. The condition of rigid bonds determines the relationship among the longitudinal and transverse displacement, $u$ and $v$, of the atoms (see Fig. 1). Going to the continuum approximation in the system lagrangian (justified by the relative weakness of the intermolecular interaction) and neglecting the dispersion and nonlinearity owing to the intramolecular interactions (a more accurate continuum description can be found elsewhere [14], we obtain a sine-Gordon equation for the longitudinal displacement $u(z, t)$ :

$$
m u_{t t}-K u_{z z}+A\left(\frac{2 \pi}{c}\right)^{2} \sin \left(\frac{2 \pi}{c} u\right)=0 .
$$

Here $K=K_{\theta}\left(2 \tan \left(\theta_{0} / 2\right)\right)^{2}$ is the rigidity parameter of the chain, so that the sound speed $v_{s}=\sqrt{K / m}$, while $A$ characterizes the height of the barrier between two neighbouring positions of the atoms in the substrate potential (1).

The sine-Gordon equation has well known soliton solutions corresponding to vacancies:

$$
u(z, t)=\frac{c}{2 \pi} 4 \arctan \exp \left(\frac{z-v t}{L}\right),
$$

where $v$ is the soliton velocity $\left(v<v_{s}\right), L=L_{0} \sqrt{1-\left(v / v_{s}\right)^{2}}$, and $L_{0}=(c / 2 \pi) \sqrt{K / A}$ is the half width of a static defect, which is larger the more rigid the chain is relative to the substrate. For our values of the constants (Table 1), we have $v s \approx 14.76 \mathrm{~km} / \mathrm{s}$ and $L_{0} \approx 35(c / 2)$.

Therefore, a vacancy (without breaking of covalent bonds, Fig. 3a) can move along the chain with a subsonic velocity, maintaining its localization and without disrupting the crystal structure outside the region of the defect. This means that at velocities not too close to that of sound, the vacancy dynamics should be those of a soliton. (When $v \rightarrow v_{s}$ it is no longer possible to neglect either the discreteness or the intramolecular nonlinearity; solitons are narrow.) This conclusion, however, is based on a quasi-one dimensional approximation for the immobile neighbouring chains. Now our goal is to study the vacancy dynamics in a crystal where all the chains are mobile.

\section{Results of a molecular-dynamic simulation of the behaviour of vacancies in a polymer crystal}

This system is characterized by the following time scales: the transit time for sound over one chain period $\approx 1.7 \cdot 10^{-2} \mathrm{ps}$, the soliton width $\approx 0.58 \mathrm{ps}$, and the period of the oscillations of the atoms in a chain in the potential of neighbouring chains $\approx 1.82 \mathrm{ps}$. 


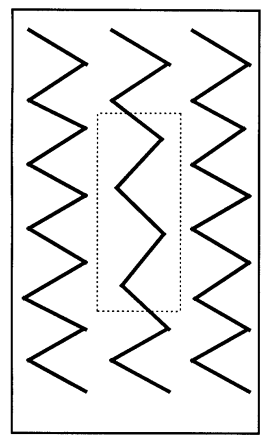

(a)

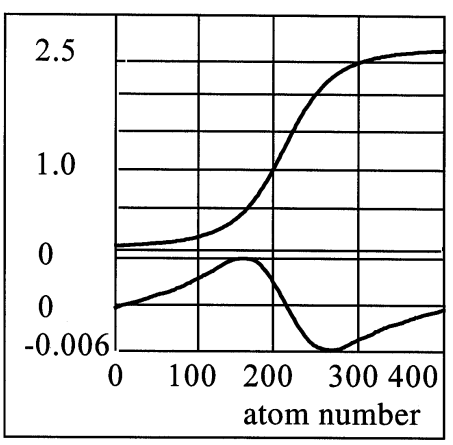

(b)
Figure 3. A vacancy in a polymer crystal chain: (a) the shape (grotesque) of the defect (the dotted box represents the defect region) (b) the logitudinal displacement of atoms from their equilibrium positions (in $\AA$ ) in molecules with a defect (upper curve) and in molecules of the first coordination sphere (lower curve).

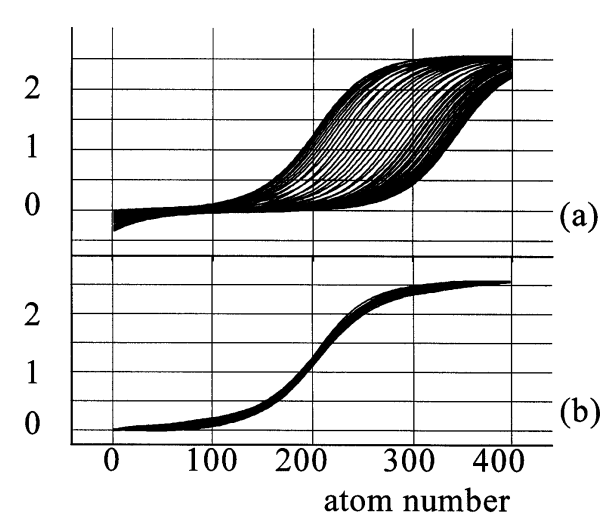

Figure 4. Sensity of static vacancies to roughness of neighbouring chains: the crystal has been heated to the temperature $0.01 K$; initial velocity of the defect is zero. Locations of the vacancy are plotted in equal time intervals (a) neighbouring chains in the crystal being immobile (b) neighbouring chains in the crystal are mobile.

In the molecular-dynamic simulation, for one of the molecules in a crystal that had relaxed and cooled to a temperature of $0.01 K$ we specified atomic displacement and velocities in accordance with the approximate analytic formula 2 and followed the evolution of the defect for a long time(on the order of hundreds of picoseconds). Almost instantly the soliton acquired a shape exactly consistent with the crystalline environment. In a sample with mobile neighbours, the presence of a vacancy in one chain causes nonuniformities to appear in all the chains within the first coordination sphere, i.e., "shadow" (see Fig. 3b) which accompany the defect even when it moves. In the numerical simulation we tracked the velocity $v_{c m}$ of the center of mass of a chain with a defect, which rescales to the vacancy velocity $v_{v a c}=-(N / 2) v_{c m}$ (where $N$ is the number of atoms in the chain).

\subsection{Difference between immobile and mobile neighbours}

Let us first heat slightly (to $0.01 \mathrm{~K}$ ) the equilibrium crystal and fix all the molecules except one (atoms having new positions deviated from the ideal ones in the crystal). Then we see that vacancies are very sensitive even to such a small "roughness" of the substrate potential: an initially static vacancy drifts very easily (of course to the location of lower energy) - see Fig. 4a. But if the neibouring chains remain mobile after heating such an extreme sensitivity disappears - Fig. 4b. We have to note, however, that at nonzero velocities dynamics of defects does not depend on either the immobile environment is ideal or it is not (for the small deviations, and large velocities).

\subsection{Type of vacancy mobility}

We now describe the results [15] of simulating the evolution of vacancies with initial velocities of $0.9,0.45$, and 0.1 times that of sound $(13.2,6.6$, and $1.5 \mathrm{~km} / \mathrm{s}$ ) in a stably equilibrium crystal (Fig. 2, frame 1). If the neighbouring molecules are fixed, defects with 


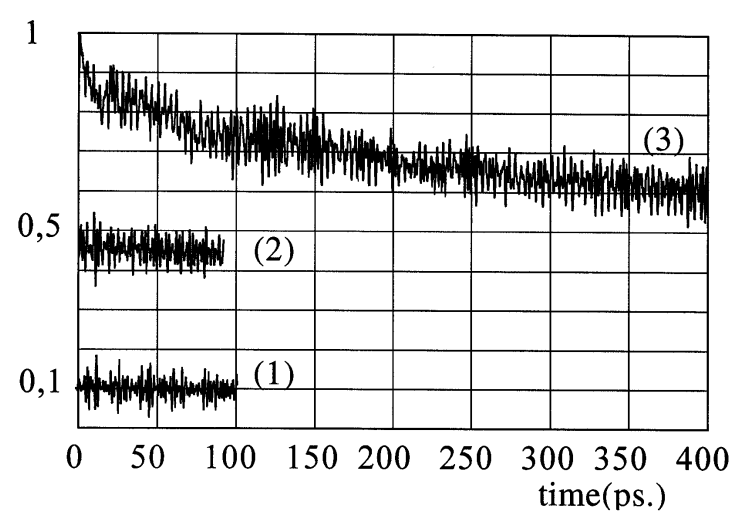

Figure 5. Vacancy dynamics in an equilibrium crystal: curves 1 and 2 illustrate the conservation of low and medium ( 0.1 and 0.45 times the sound speed) velocities of defects in a chain surrounded by mobile neighbours; curve 3 , the slowing down of fast ( 0.9 times the sound speed) defect with a transition to stationary soliton motion at a lower velocity $(\approx 0.6$ times the sound speed).

all three of these velocities move smoothly along the chain with their velocities essentially unchanged for at least 100ps. Over this time, they can cover 5186, 2593, and 576 chain periods, respectively. When all the molecules are mobile, the dynamics of the two slowest defects is the same (Fig. 5). Only for the fastest defect does the velocity fall off slowly, decreasing to $\approx 0.6$ times the sound speed $(9 \mathrm{~km} / \mathrm{s})$ over $400 \mathrm{ps}$. Therefore, the mobility of the surrounding chains does not affect the behaviour of vacancies moving at low and medium velocities, but slows down the faster defects to medium velocities without changing their soliton-type dynamics.

This slowing down is not caused by the radiation of energy by the defect into chain along which it moves owing to effects of the discreteness which show up at higher defect velocities [7]: for $v=0.9 v_{s}$ the half width $L$ of the solitons is still quite large $L \approx 15(c / 2)$ (Eq. (2)). Evidently, the reason for the slowing down of fast defects in a crystal is their more intense interaction with the mobile molecules of neighbouring chains. This effects has not been observed before and requires separate theoretical and numerical study.

\subsection{Influence of crystalline structure on type of vacancy mobility}

Note that the configuration of the crystalline environment has no effect on the character of the dynamics of a defect in a chain if the neighbouring chains are immobile, but changes it fundamentally when the neighbouring chains are mobile. Specifically, in nonequilibrium or unstable structures (e.g., a nonequilibrium orthorhombic structure in the united atom model of polyethylene [6] or an unstable monoclinic structure of Fig. 2, frame 2) which require rotation of chains for relaxation, a vacancy will be slowed down rapidly.

For example, in the latter case a vacancy with an initial speed of $4.6 \mathrm{~km} / \mathrm{s}$ is stopped over a time on the order of $10 \mathrm{ps}$, having covered about 100 chain periods. The onset of this kind of slowing down was apparently observed in Ref. [6] and served as the basis for the unjustified claim that the twisting defect dynamics did not exhibit soliton behaviour.

In a molecule at the boundary between two domains in a relaxed stratifying sample (Fig. 2, frame 2), point defects are also slowed down and change the structure of the boundary between the domains. 


\section{Summary}

- In an equilubrium polymer crystal, vacancies caused by localized tensile deformation of the chain have a soliton-type mobility. Soliton-like excitations conserve their low and medium velocities. Initially fast defects slow down with a transition to a stationary motion at a lower velocity, energy going into the neighbouring chains.

- In an nonequilibrium polymer crystal, mobility of vacancies is non-solitonic.

- Mobility of neighbouring chains has a stabilizing effect on static soliton-like excitations: their extreme sensitivity to small deviations of atoms in neibouring chains from their ideal positions disappears.

\section{Acknowledgements}

This work was supported by the Russian Foundation for Basic Research (Grant 98-0333366a). One of the authors (EZ) is very grateful to the Organizing Committee of the Conference and Russian Foundation for Basic Research for their financial support of her participating in the Conference.

\section{References}

[1] Ginsburg V V, Manevitch L I and Ryvkina N G, Mekhanika Kompozitnykh Materialov, 1991, N 2, 249 (in Russian).

[2] Ginsburg V V and Manevitch L I, Fiz. Tverd. Tela, 1990, V.32, 2414 (Sov. Phys. Solid State, 1990, V.32, 1401).

[3] Boyd R H, Polymer, 1985, V.26, 323.

[4] Boyd R H, Polymer, 1985, V.26, 1123.

[5] Kimming M, Strobl G and Stuhn B, Macromolecules, 1994, V.27, 2481.

[6] Noid D W, Sumpter B G and Wunderlich B, Macromolecules, 1991, V.24, 4148.

[7] Peyrard M and Kruskal M D, Physica D, 1984, V.14, 88.

[8] Peyrard M and Remoissenet M, Phys. Rev. B, 1982, V.26, 2886.

[9] Balabaev N K, Gendelman O V, Mazo M A and Manevitch L I, Zh. Fiz. Khim., 1995, V.69, 24 (in Russian).

[10] Noid D W, Sumpter B G and Wunderlich B, Macromolecules, 1990, V.23, 664.

[11] Rigby D and Roe R J, Macromolecules, 1989, V.22, 2259.

[12] Allen M P and Tildesley P J, Computer Simulation of Liquids, Clarendon Press, Oxford, 1987.

[13] Khalatur P G, Balabaev N K and Pavlov A S, Molec. Phys., 1986, V.59, 753.

[14] Manevitch L I and Savin A V, Phys. Rev. E, 1997, V.55, 4713.

[15] Zubova E A, Balabaev N K and Manevitch L I, Zh. Exp. Teor. Fiz., 1999, V.115, 1063 (J. Exper. and Theor. Phys., 1999, V.88, 586). 